\title{
Estilos de aprendizaje en estudiantes de inglés y su rendimiento académico*
}

\section{Students learning styles in English and academic performance}

\section{Alunos estilos $\in$ Inglês $\in$ desempenho acadêmico de aprendizagem}

\author{
Myriam Cabrales Vargas, Universidad de San Buenaventura, \\ Cartagena, Colombia** \\ Martha Oliveros Torres, Universidad de San Buenaventura, \\ Cartagena, Colombia*** \\ Alicia Mejía López, Universidad de San Buenaventura, \\ Cartagena, Colombia****
}

ReCibido: 2 de AGOSTO DE 2013 - Aprobado: 10 DE FEBRERo de 2014

Para citar este artículo: Cabrales, M., Oliveros, M. y Mejía, A. (2014).

Estilos de aprendizaje en estudiantes de inglés y su rendimiento académico. Itinerario Educativo, XXVIII (63), 155-177

* Artículo de investigación derivado del proyecto Estilos de aprendizaje y rendimiento académico en inglés en estudiantes de la Licenciatura en Lenguas Modernas de la Universidad de San Buenaventura, realizada entre junio de 2011 y septiembre de 2012 en la Universidad de San Buenaventura, seccional Cartagena. Grupo Interdisciplinario de Investigación en Educación y Pedagogía.

** Master en Ciencias del Lenguaje (Universidad de Franche-Comté), directora de la Licenciatura en Lenguas Modernas de la Universidad de San Buenaventura, seccional Cartagena, Colombia.E-mail: mcabrales@usbctg.edu.co

*** Psicóloga (Universidad del Norte), maestrante en Educación (Universidad de Cartagena), docente de la Universidad de San Buenaventura, seccional Cartagena, Colombia. E-mail: moliveros@usbctg.edu.co

**** Especialista en Enseñanza del inglés y Magíster en educación con énfasis en inglés (Universidad del Norte). Docente de la Universidad de San Buenaventura, seccional Cartagena, Colombia.E-mail: amejia@usbctg.edu.co 
Resumen. Este artículo constituye la síntesis de una investigación que se propuso conocer los estilos de aprendizaje de los estudiantes de la Licenciatura en Lenguas Modernas de la Universidad de San Buenaventura, específicamente en el área de inglés, mediante la aplicación del test de Felder y Silverman. Además, se buscó contrastar esa información, con el rendimiento académico de los investigados, expresado en el promedio de notas y en la opinión de los docentes, obtenida mediante la técnica del grupo focal. Los resultados indican un predominio de los estilos activo, sensitivo, visual y secuencial, frente a los estilos reflexivo, intuitivo, verbal y global. En relación con el rendimiento académico, se encontró que éste es superior en los estudiantes que poseen en alguna medida, una mayor dosis de los estilos de la segunda tendencia (reflexivo, intuitivo, verbal y global). Por otra parte, las notas cuantitativas, no reflejan una diferencia muy amplia entre los niveles calificados cualitativamente como: bueno, regular y bajo, para cuya descripción los docentes fueron más exigentes de lo que reflejan las notas o calificaciones. La sugerencia de las investigadoras se orienta a la necesidad de propiciar un mayor equilibrio entre las dos categorías bipolares de los estilos de aprendizaje, además, a revisar los criterios y formas de evaluación.

Palabras clave. Estilos de aprendizaje, estilo activo, estilo reflexivo, estilo sensitivo, estilo visual, estilo verbal, estilo secuencial, estilo verbal, rendimiento académico, personalidad, actitud, capacidades mentales (Tesauro Unesco).

Abstract. This article is the synopsis of a program of research aimed at exploring the learning styles of the students of the BA in Modern Languages at Universidad de San Buenaventura, by using the test method of Felder and Silverman. In addition, we sought to compare the resulting information with the academic performance of those investigated, expressed in average grades and in the opinions of the teachers, obtained by the focus group technique. The results indicate a predominance of active, sensory, visual and sequential styles versus reflective, intuitive, verbal and global styles. In relation to academic achievements, we found that it is higher in students who have to some extent, a higher utilization of the second trend styles (reflective, intuitive, verbal and global). Moreover, quantitative notes do not show how much difference from the quantitatively rated levels of good, fair and low, for which the disclosure of the teachers were more demanding of what grades reflect. The preliminary conclusion of the research is to focus on the need to promote a 
better balance between the two alternate categories of learning styles. The research also suggests to review the criteria and evaluation forms.

Keywords. Learning style, active learning style, reflexive learning style, sensitive learning style, visual learning style, verbal learning style, sequential learning style, global learning style academic performance, personality, attitude, mental abilities (Unesco Thesaurus).

Resumo. Este artigo é a síntese de uma investigação é conhecer os estilos de aprendizagem dos alunos da Licenciatura em Línguas Modernas da Universidade de San Buenaventura, especificamente na área de Inglês, através da aplicação do teste de Felder e Silverman. Além disso, buscou-se comparar essa informação com o desempenho acadêmico dos investigados, expressa em média classificações e opiniões de professores, obtidas pela técnica de grupo focal. Os resultados indicam uma predominância de estilos sensíveis, visuais e seqüenciais ativos em relação ao estilo reflexivo, intuitivo, verbal e global. Com relação ao desempenho acadêmico, descobrimos que este é maior em alunos que possuem algum grau, uma dose mais elevada do segundo estilos de tendência (reflexivo, intuitivo, verbal e global). Além disso, as avaliações quantitativas não refletem uma diferença muito grande entre os níveis qualitativamente e qualificados: bom, média e baixa para os professores de divulgação eram mais exigente do que está graus refletir. A sugestão tem por objetivo investigar a necessidade de promover um melhor equilíbrio entre as duas categorias bipolares de estilos de aprendizagem também para rever os critérios e formas de avaliação.

Palavras-chave. Estilos aprendizagem, estilo ativo, estilo reflexivo, estilo sensível, estilo visual, o estilo verbal, estilo sequencial, o estilo verbal, desempenho acadêmico, personalidade, atitude, habilidades mentais (Unesco Thesaurus).

\section{Introducción}

Indagar acerca del estilo de aprendizaje de los estudiantes, es una necesidad de todo programa de formación, si la meta es precisamente que éstos obtengan buenos resultados. Éste fue el propósito que orientó la realización de la investigación aquí expuesta, ya que el análisis de las notas obtenidas en inglés, el índice de repetición de las asignaturas de esa área y el concepto de los docentes sobre el bajo compromiso 
de muchos estudiantes con el estudio, representaba una problemática que requería soluciones. El objetivo central del proyecto fue entonces conocer los estilos de aprendizaje de los estudiantes, compararlos con su rendimiento académico en inglés y establecer alguna relación entre estas dos variables. En relación con la segunda variable, no sólo se hizo el levantamiento del promedio de las notas de los estudiantes, sino que también se indagó sobre el concepto de los docentes en relación con las categorías psicológicas o internas de personalidad, actitud e inteligencia. Con base en los resultados de este diagnóstico, se podrá poner en marcha - en el futuro - un plan de intervención docente que genere beneficios a los estudiantes en su proceso de aprendizaje del inglés, lo que se constituye en el impacto esperado.

\section{Referentes teóricos}

\section{Aprendizaje}

Seleccionar un concepto de aprendizaje implica realizar una compleja tarea de revisión bibliográfica en el área de la psicología. El resultado confirmará que generalmente la mayoría de las teorías psicológicas del aprendizaje, como lo señala Pérez (1992), "son modelos explicativos que han sido obtenidos en situaciones experimentales, y hacen referencia a aprendizajes de laboratorio que sólo relativamente pueden explicar el funcionamiento red de los procesos naturales del aprendizaje incidental y del aprendizaje en el aula" (p. 36). Por tal motivo, este autor considera necesario tener en cuenta tanto los procesos internos que se dan en el individuo que aprende, como la relación de éste con el entorno físico y sociocultural, y define el aprendizaje como "los procesos subjetivos de captación, incorporación, retención y utilización de la información que el individuo recibe en su intercambio continuo con el medio" (Pérez, 1988, citado en García Cué, 2006) ${ }^{1}$. Concepto al que se acogió la investigación que aquí se presenta, precisamente por el acercamiento que era necesario hacer entre la teoría, las situaciones naturales y propias del aula y las actividades que debe desarrollar el estudiante en su proceso de aprendizaje. Además, este concepto logra una conexión adecuada con las teorías sobre estilos de aprendizaje, que precisamente tratan de explicar, de qué manera el individuo activa los procesos subjetivos frente a la información proveniente de su relación, con el medio externo físico o sociocultural.

1 Texto electrónico sin paginación 


\section{Estilos de aprendizaje}

Como concepto de estilos de aprendizaje, se consideró pertinente el que postula que "los estilos de aprendizaje son rasgos cognitivos, afectivos y fisiológicos que sirven como indicadores relativamente estables, de cómo los alumnos perciben interacciones y responden a sus ambientes de aprendizaje" (Keefe, 1988, citado en Terrádez, 2007, p. 227). Este concepto está enmarcado en el ámbito escolar, además sugiere que cada estudiante tiene su propio estilo de aprendizaje, en función de los rasgos propuestos por el autor.

En relación con las distintas formas de concebir los estilos de aprendizaje, es decir "las preferencias o tendencias a utilizar más unas determinadas maneras de aprender que otras". (Arvizu, 2010, p. 6), una detenida revisión de la bibliografía propuesta sobre el tema, permitió identificar las teorías más reconocidas, de las cuales las más relevantes para este estudio, porque enfatizan en la manera como el sujeto se relaciona con la información son:

El modelo de aprendizaje basado en experiencias ${ }^{2}$ que propone cuatro estilos de aprendizaje: activo, reflexivo, teórico y pragmático, que corresponden a la forma como el sujeto se relaciona con la información. Si lo hace de manera directa y concreta es de estilo activo; si lo hace de manera abstracta, es teórico; si la información se transforma en conocimiento cuando se piensa y se reflexiona, se trata de un aprendizaje de tipo reflexivo, y cuando se requiere experimentar con la información en forma activa, entonces se tiene un estilo de aprendizaje pragmático (Cisneros, 2004, pp. 22-29).

El modelo de Programación Neurolingüística (PNL), elaborado a principios de los 70' por Bandler y Grinder (1988), citados en Cisneros (2004, pp. 30-34), es también conocido por la sigla VAK porque se basa en el sistema utilizado para representar la información recibida, que puede ser: visual, auditivo y kinestésico. Los alumnos visuales son aquellos que necesitan ver la información que van a aprender, para facilitar el aprendizaje de conceptos abstractos; además, les gusta planear sus actividades con anterioridad. Los alumnos auditivos aprenden más fácilmente cuando se les presenta de manera oral y ordenada

2 Elaborado por Kolb en los años setenta y divulgado en 1984. 
la información; poseen habilidades no sólo para explicar a otros la información que escuchan, sino también para explicársela a sí mismos. El estudiante kinestésico prefiere experimentar y poner en práctica el aprendizaje por medio de sensaciones y movimientos

El modelo de los cuadrantes cerebrales ${ }^{3}$ basado en el funcionamiento del cerebro, plantea que los cuatro cuadrantes, en donde se entrecruzan los hemisferios izquierdo y derecho, y los cerebros límbicos y corticales, determinan el estilo de aprendizaje, según la dominancia que ejerzan cada uno de ellos. Cada cuadrante cerebral opera, piensa, crea, aprende, suma y se relaciona con el mundo de manera diferente Se dice entonces que el alumno cortical-izquierdo se rige por pruebas y hechos y posee una buena capacidad de análisis y razonamiento. Al límbico-izquierdo le gustan las clases metódicas y planificadas pues le ayudan a integrar sus conceptos. Para el límbico-derecho, las relaciones sociales y la empatía con el profesor son fundamentales para lograr su aprendizaje; como ser social, necesita compartir su aprendizaje para interiorizarlo. Por último, el cortical-derecho se caracteriza por seleccionar sólo aquello que encuentra esencial para aprender, por ello tiende a dispersarse y a mostrar problemas para planificar su trabajo (Cisneros, 2004, pp. 7-19).

El modelo de las categorías bipolares, desarrollado por Richard Felder y Linda Silverman, en 1988, considera cinco categorías cada una de las cuales corresponde a dos polos opuestos. Se trata de un modelo mixto que incluye algunos estilos de aprendizaje de los otros modelos ya descritos, por lo cual se consideró pertinente adoptarlo para el estudio al que se refiere este artículo, ya que permite indagar sobre qué tipo de información percibe preferentemente el estudiante (sensitivo/ intuitivo), cómo la percibe o se la representa más efectivamente (visual/ verbal), cómo la procesa (activo/reflexivo) y cómo la organiza para comprenderla mejor (secuencial/ global). Además, plantea una quinta categoría, relativa al tipo de organización de la información con la que se siente más cómodo el estudiante (inductivo/ deductivo), la cual no fue tenida en cuenta en esta investigación, porque se trata de una cualidad de la información misma, más no del proceso de aprendizaje (Cisneros, 2004, pp. 20-21).

3 Modelo desarrollado en 1976 por Ned Herrmann, conocido en inglés como el Herrmann Brain Dominance Instrument. 
Como lo explica Cisneros (2004, p. 21), los estudiantes sensitivos son concretos y prácticos y prefieren basarse en hechos y procedimientos pre-establecidos, para resolver problemas. Son pacientes frente a explicaciones detalladas; les gusta el trabajo que requiera de la práctica y memorizan hechos con facilidad. No se sienten cómodos en los cursos teóricos, que no tengan conexión evidente con el mundo que los rodea, además, "les disgustan las complicaciones y las sorpresas" (Salas, 2008, p. 70). Los intuitivos por su parte disfrutan creando, innovando y descubriendo, por ello rechazan las repeticiones y dan la bienvenida al nuevo aprendizaje. Las teorías, las matemáticas y las abstracciones son su fuerte, mas no gustan de la memorización o cálculos de rutina. "Pueden ser mejores para captar nuevos conceptos y a menudo se sienten más cómodos que los sensitivos con las abstracciones y con las fórmulas matemáticas" (Salas, 2008, pp. 70-71).

Los visuales prefieren la información que contenga ilustración gráfica: diagramas de flujo, películas, imágenes, figuras, etc. Recuerdan mejor lo que ven pues es factible que "si algo simplemente se dice y no se muestra, hay una gran probabilidad de que no lo retengan". (Salas, 2008, p. 71). Los verbales se guían por la información hablada o escrita y recuerdan mejor lo que leen o lo que oyen; sin embargo, "cada cual aprende mejor cuando la información es presentada de manera tanto visual como verbal" (Salas, 2008, p. 71).

Los alumnos activos necesitan hacer algo con la información para poder aprenderla. Disfrutan el trabajo en grupo y se les dificulta aprender, si no realizan alguna actividad con la información: aplicarla, explicarla, discutirla, transformarla. A los reflexivos, por su parte, se les facilita el aprendizaje cuando se les da la oportunidad de reflexionar y pensar sobre la nueva información. Prefieren aprender meditando tranquilamente, pensando y trabajando solos. "También se inclinan por aprender de materiales presentados ordenadamente a través de libros de trabajo, conferencias, y demostraciones" (Guanipa y Mogollón, 2006, p. 16).

Los secuenciales aprenden paso a paso. Cada etapa o parte de la información, debe estar bien relacionada con la anterior de manera lógica y ordenada; son amantes del orden por naturaleza. Los globales, por el contrario, aprenden a "grandes saltos", pueden visualizar fácilmente el todo; pueden resolver problemas complejos rápidamente; sin embargo, 
pueden presentar dificultades al tratar de explicar cómo lo hicieron (Cisneros, 2004, p. 21).

Aunque el binomio inductivo/deductivo no hace parte del estudio realizado, por su relación con las categorías anteriores, conviene recordar que el primer tipo corresponde a quienes prefieren conocer primero los hechos y particularidades; y el segundo a quienes precisan conocer primero los fundamentos generales para luego deducir o inferir las particularidades. Según Salas (2008), “existen considerables pruebas de que el incorporar un sustancial componente inductivo en la enseñanza promueve un aprendizaje efectivo (p. 71).

Cabe finalmente resaltar, que Felder planteó que las categorías de este modelo no son absolutas, lo que explica Salas (2008) al expresar que "las preferencias de los estudiantes, de acuerdo con las cinco escalas que se mencionan anteriormente, pueden variar desde moderadas a fuertes, o simplemente dejar de existir con el tiempo, o con el tópico de estudio o ambiente de aprendizaje" (p. 70).

\section{Rendimiento académico}

El rendimiento académico (RA), es definido por Pizarro (1985) "como una medida indicativa de las capacidades respondientes que manifiestan, en forma estimativa, lo que una persona ha aprendido como consecuencia de un proceso de instrucción o formación" (citado por Alcaide, 2009, p. 31). Confirmando la cualidad de medición, implícita en esta variable, Alcaide (2009) relaciona el RA con la normativa educativa vigente y expresa que éste "se define como el proceso alcanzado por los alumnos, en función de los objetivos programáticos y que puede ser medido, mediante la realización de actividades de evaluación" (p. 32).

Las anteriores conceptualizaciones, condujeron a considerar que para este estudio era fundamental, tener en cuenta los resultados numéricos de la evaluación, es decir las notas o calificaciones, obtenidas por los estudiantes en inglés. Sin embargo, teniendo en cuenta la diversidad de variables de carácter social (familia, contexto), biológico (sexo, edad), académico (docentes, metodologías, recursos) o psicológico (motivación, actitud, inteligencia, personalidad), que intervienen en el RA, también era necesario tener en cuenta al menos una de estas dimensiones, por lo que se optó por la variable psicológica o interna, expresada en las 
categorías de personalidad, actitud e inteligencia. En este estudio, esas categorías corresponden brevemente a los siguientes conceptos:

Personalidad: Característica psíquica cuya conceptualización es compleja, debido a las diversas teorías existentes para tratar de explicarla (dinámica, de los rasgos, del desarrollo, entre otras). Sin embargo, de manera general, se puede definir como las causas internas que subyacen al comportamiento individual y a la experiencia de una persona (Clonninger, 2002).

Actitud: Conjunto de predisposiciones a responder, a alguna clase de estímulo, con ciertas clases de respuestas. Más específicamente, se trata de una disposición aprendida a responder de una manera consistentemente favorable o desfavorable a un objeto dado (objeto físico, personas, grupos, etc.). Es un concepto multidimensional cuyos componentes son: el cognitivo, el afectivo y el conductual (Ovejero, 2007).

Inteligencia: Capacidad mental muy general que, entre otras cosas, implica la habilidad de razonar, planear, resolver problemas, pensar de manera abstracta, comprender ideas complejas, aprender rápidamente y aprender de la experiencia. No es un mero aprendizaje de los libros, ni una habilidad estrictamente académica, ni un talento para superar pruebas, más bien, el concepto se refiere a la capacidad de comprender el propio entorno. (Gottfredson, 2004).

\section{Antecedentes investigativos}

Aun cuando son numerosos los estudios realizados sobre esta temática, para esta investigación se tuvieron en cuenta como antecedentes, principalmente aquellos que se refieren al área de las lenguas y en particular a las lenguas extranjeras. Se referencian a continuación, solamente cuatro de esos estudios, por considerarlos los más pertinentes:

“Los estilos de aprendizaje: una propuesta pedagógica para optimizar la enseñanza de las lenguas extranjeras en la Licenciatura en Lenguas Modernas de la Universidad del Quindío" (Loaiza y Guevara, 2012). Investigación de corte transversal y exploratorio-descriptivo, basada en la aplicación del modelo de Felder y Silverman, para identificar los estilos de aprendizaje de estudiantes y del modelo de Herrmann para conocer el estilo personal de enseñanza de profesores de los cursos 
básicos de inglés y francés. Los resultados señalan la heterogeneidad en los estilos de aprendizaje de los estudiantes y la disimilitud entre esos estilos y el estilo personal de enseñanza de los docentes. Con base en esos resultados, los autores proponen algunos lineamientos para la posterior estructuración de una propuesta pedagógico-didáctica que optimice la enseñanza y el aprendizaje de las lenguas extranjeras y que sirva de guía para el diseño curricular que tenga en cuenta los estilos de los actores en estudio.

"Estilos y Estrategias de Aprendizaje en el rendimiento académico de los alumnos del área de Inglés de la Licenciatura en Lenguas Modernas de la Universidad de La Salle" (Hernández y Cardona, 2008). Estudio experimental que indagó sobre el impacto que tiene sobre el rendimiento académico el enseñar según los estilos de aprendizaje auditivo, visual y kinestésico y las estrategias que mejor los complementan. Los resultados determinaron que el grupo experimental superó en porcentaje de 7.6 al de control. Además, teniendo en cuenta el número de alumnos que pasaron las pruebas con puntajes superiores a 30/50, se encontró que en el grupo experimental un $39.5 \%$ más de alumnos lo lograron, mientras que en el de control solo el $17.8 \%$ más lo hicieron. Esto evidencia una diferencia de $21.7 \%$ a favor del grupo experimental. Finalmente, los autores concluyeron que una metodología para la enseñanza del inglés como lengua extranjera, que tenga en cuenta la forma como aprenden los estudiantes e involucre estrategias de aprendizaje acordes con cada estilo de aprendizaje, conduce a un mejor rendimiento académico.

"La importancia de los estilos de aprendizaje en la enseñanza de inglés como lengua extranjera" (Hernández, 2004). Investigación de carácter exploratorio-descriptivo sobre los estilos de aprendizaje cognoscitivos, sensoriales y afectivos, predominantes en una población representativa de estudiantes de inglés del Centro de Enseñanza de Lenguas Extranjeras (CELE) de la Universidad Nacional Autónoma de México (UNAM). Con base en los resultados de tres tests diferentes, la autora logra probar sus dos hipótesis, a saber: 1) Existen diferencias en los estilos de aprendizaje predominantes de los estudiantes universitarios y 2) Hay una relación entre estilos de aprendizaje predominantes y la edad, el género, el nivel de escolaridad y el área de estudio de los estudiantes.

Los resultados de esa investigación mostraron que el estilo cinestético predomina entre estudiantes de ambos sexos, de licenciatura o maestría, 
de 17 a 32 años de edad; pero, se da un mayor predominio del hemisferio izquierdo del cerebro, si son de género femenino y tienen entre 17 y 24 años.

El predominio de dependencia o independencia de campo, del estilo cognoscitivo, resultó ser propio de estudiantes de edades entre los 33 y 40 años. Entre las sugerencias formuladas, la autora expresa la necesidad de comprender mejor los estilos de aprendizaje y que tanto docentes como instituciones amplíen el abanico de posibilidades de los materiales y la metodología en el aula.

“Estilos de aprendizaje, estrategias de lectura y su relación con el rendimiento académico de la lengua extranjera" (Manzano e Hidalgo, 2009). Investigación realizada con el objetivo de conocer la relación entre los estilos reflexivo, activo, teórico y pragmático, el uso de estrategias de lectura y el rendimiento en estudiantes de lengua inglesa, de diversas carreras de la Universidad de Ciego de Ávila (Cuba). El empleo del test $\mathrm{CHAEA}^{4}$ y de un cuestionario de estrategias de lectura, validado por los autores, permitió determinar lo siguiente:

El estilo reflexivo muestra una tendencia a un predominio más alto, mientras mayor es la evaluación en el nivel de dominio de la lengua extranjera; el estilo activo, por el contrario, muestra una tendencia a un predominio más bajo (...). Mientras más alto es el domino de la lengua, más alta es la frecuencia del uso de estrategias de lectura (Manzano e Hidalgo, 2009, p. 147).

Los autores además señalan, entre muchas otras, como causas del bajo rendimiento la deficiencia en la explotación adecuada de los procesos de lectura en la enseñanza del inglés, pues la comprensión de textos se orienta hacia la decodificación y no hacia la construcción de significados; además, la tendencia a dirigir la enseñanza-aprendizaje al resultado y no al proceso lógico y racional que éste conlleva.

En contraste con los anteriores estudios, la investigación que aquí se presenta, hace referencia únicamente a las dos variables antes mencionadas: los estilos de aprendizaje y el rendimiento académico, enmarcadas en los siguientes conceptos.

4 CHAEA es la sigla de: Cuestionario de Honey Alonso sobre Estilos de Aprendizaje. 


\section{Metodología}

\section{Enfoque y tipo de investigación}

La investigación se realizó conjugando las perspectivas de los enfoques cuantitativo y cualitativo. En relación con el primero, en cuanto se indagó sobre datos precisos, obtenidos a través de un test ${ }^{5}$ aplicado a los estudiantes, cuyos resultados son presentados en forma de porcentajes. El carácter cualitativo está dado tanto por la naturaleza misma del objeto de estudio (un grupo de seres humanos) como por las diversas posibilidades de interpretar las realidades y experiencias, por parte de los investigadores y de los investigados (docentes y estudiantes). Por consiguiente, no se trata de buscar una verdad objetiva y absoluta, sino de configurar sentidos y comprender los significados que las personas investigadas dan a las realidades que viven. Así, los estudiantes expresaron sus percepciones en relación con sus estilos de aprendizaje y los docentes sobre el rendimiento académico de éstos.

Se trata específicamente de una investigación de tipo descriptivointerpretativo porque caracteriza los estilos de aprendizaje de los estudiantes, lo cual adquiere sentido a través de la interpretación que de ellos hacen las investigadoras, a la luz de las teorías de los autores en que se fundamentó la investigación. Tiene además un componente comparativo porque se buscó también establecer qué diferencias en rendimiento académico presentan los estudiantes según sus distintos estilos de aprendizaje.

\section{Variables y categorías de análisis}

Como se expresó anteriormente, las dos variables en estudio fueron: los estilos de aprendizaje y el rendimiento académico en inglés. En relación con las categorías, para la primera variable se tuvo en cuenta la propuesta de Felder y Silverman, ya presentada en la introducción de este artículo que sugiere los estilos en paralelos bipolares, así: sensitivo-intuitivo, visuales-verbales, inductivo-deductivo, activo-reflexivo y secuencialesglobales. Para la segunda variable, se consideraron: el promedio obtenido

5 Cuestionario de Felder y Silverman. OVEDESA: Observatorio vocacional de estudios y diagnóstico de estilos de aprendizaje. Disponible en: http://artesycienciasatenea. org/odunicor/ovedesa2/reportes/rep_test_felder_preguntas.php 
en inglés los tres semestres anteriores a la investigación y tres de los elementos psicológicos intervinientes en el rendimiento académico: personalidad, actitud e inteligencia.

\section{Recolección y procesamiento de la información}

La recolección de la información se hizo mediante las siguientes técnicas e instrumentos:

- $\quad$ Encuesta: Cuestionario, según el test propuesto por Felder y Silverman, para identificar el estilo de aprendizaje, aplicado a una muestra de 48 estudiantes de VI a X semestre del programa de Lenguas Modernas de la Universidad de San Buenaventura, seccional Cartagena ${ }^{6}$

- Entrevista (grupo focal): Orientada a conocer de manera directa el concepto de los cuatro docentes de inglés, sobre el rendimiento académico de los estudiantes bajo un calificativo general (bueno, regular, bajo) y de manera particular su apreciación en relación con la personalidad, la actitud y la inteligencia de los mismos

- Levantamiento de datos del portal académico, relativos a las notas y promedio de los tres últimos semestres, anteriores a la investigación.

Los datos arrojados por el test permitieron determinar el estilo de aprendizaje de cada estudiante en particular. Sin embargo, la interpretaciónx se hace de manera general, con base en los porcentajes que cada calificación representa respecto a la totalidad del grupo.

Las interpretaciones tanto de los resultados de la encuesta, como del grupo focal y el promedio de los estudiantes en inglés están acompañadas de explicaciones de los fenómenos encontrados. Estas explicaciones están sustentadas en el deber ser, con base en los principios que se establecieron en el marco teórico, así como en la experiencia y visión de las investigadoras sobre el ejercicio del aprendizaje y el rendimiento académico esperado.

6 Jóvenes de clase media, pertenecientes en su mayoría a los estratos 2 y 3, de edades que oscilan por lo general entre los 17 y 24 años. 


\section{Resultados}

La Tabla 1 presenta el número de estudiantes por semestre, según los indicadores cualitativos de rendimiento académico (bueno, regular, bajo) sugeridos por los docentes. Cada indicador fue definido por éstos en la discusión del grupo focal, información que se presenta en anexo, al final del artículo. Las columnas de las notas presentan el rango de las mismas (la más alta y la más baja) y el promedio del grupo en la parte inferior.

Tabla 1:

Rendimiento académico

\begin{tabular}{|c|c|c|c|c|c|c|c|}
\hline \multirow[t]{2}{*}{ Sem } & \multicolumn{7}{|c|}{ Rendimiento académico } \\
\hline & Bueno & Notas & Regular & Notas & Bajo & Notas & $\begin{array}{l}\text { Total } \\
\text { est. }\end{array}$ \\
\hline \multirow{2}{*}{$10^{\circ}$. } & \multirow{2}{*}{4} & $4.3-4.7$ & & & & & \multirow{2}{*}{$\begin{array}{c}4 \\
8.3 \%\end{array}$} \\
\hline & & 4.52 & & & & & \\
\hline \multirow{2}{*}{$9^{\circ}$. } & \multirow{2}{*}{5} & $4.3-4.9$ & \multirow{2}{*}{1} & 4.4 & \multirow{2}{*}{2} & $3.8-3.9$ & \multirow{2}{*}{$\begin{array}{c}8 \\
16.6 \%\end{array}$} \\
\hline & & 4.56 & & 4.4 & & 3.85 & \\
\hline \multirow{2}{*}{$8^{\circ}$} & \multirow[b]{2}{*}{5} & $4.1-4.6$ & \multirow{2}{*}{7} & $3.7-44$ & \multirow{2}{*}{2} & $3.9-4.1$ & \multirow{2}{*}{$\begin{array}{c}14 \\
29.16 \%\end{array}$} \\
\hline & & 4.32 & & 4.1 & & 4.0 & \\
\hline \multirow{2}{*}{$7^{\circ}$} & \multirow{2}{*}{3} & $3.8-4.4$ & \multirow{2}{*}{1} & 4.2 & \multirow{2}{*}{4} & $2.6-33$ & \multirow{2}{*}{$\begin{array}{c}8 \\
8.3 \% \\
\end{array}$} \\
\hline & & 4.13 & & 4.2 & & 3.07 & \\
\hline \multirow{2}{*}{$6^{\circ}$} & \multirow{2}{*}{4} & $3.8-4.1$ & \multirow{2}{*}{4} & $3.5-4.1$ & \multirow{2}{*}{1} & 3.8 & \multirow{2}{*}{$\begin{array}{c}9 \\
18.75 \%\end{array}$} \\
\hline & & 3.95 & & 3.72 & & 3.8 & \\
\hline \multirow{2}{*}{$5^{\circ}$} & & & \multirow{2}{*}{4} & $3.3-3.9$ & \multirow{2}{*}{1} & 3.3 & \multirow{2}{*}{$\begin{array}{c}5 \\
10.41 \%\end{array}$} \\
\hline & & & & 3.57 & & 3.3 & \\
\hline \multirow{2}{*}{ Total } & 21 & \multirow{2}{*}{4.29} & 17 & \multirow{2}{*}{3.99} & 10 & \multirow{2}{*}{3.6} & 48 \\
\hline & $43.7 \%$ & & $35.4 \%$ & & $20.8 \%$ & & $100 \%$ \\
\hline
\end{tabular}

El análisis de los datos contenidos en la Tabla 1, interpretados desde los enfoques cuantitativo y cualitativo, sugieren las siguientes apreciaciones:

La diferencia en el promedio general de las notas entre los niveles bueno (4.29), regular (3.99) y bajo (3.6), no es tan grande como se pudiera esperar, ya que en este caso sólo alcanzó las tres décimas, entre el primero y el segundo nivel y cuatro entre éste y el último. Esta cercanía en los 
promedios de notas cuantitativas, es producida por el hecho de que haya estudiantes calificados cualitativamente como buenos, a pesar de tener notas muy próximas a los que fueron calificados como regulares. Por ejemplo, en ambas categorías se encuentran estudiantes con promedios de 4.1 a 4.4 , notas que suben el promedio general de los regulares y bajan el de los buenos.

El anterior resultado permite inferir que para los docentes la nota no es un indicador determinante, para considerar que un estudiante es bueno, regular o malo. Al momento de adjudicar tales calificativos, con base en la discusión en la entrevista en grupo focal, las consideraciones sobre la personalidad, la actitud y las capacidades intelectuales, fueron más significativas. Podría entonces inferirse que las evaluaciones expresadas en las notas cuantitativas, ingresadas al sistema de Registro Académico, no expresan en su totalidad las cualidades de los estudiantes en lo que se refiere a sus características personales, la actitud asumida en el aula frente a las actividades desarrolladas o a los trabajos fuera de ésta, ni a sus capacidades intelectuales.

La anterior interpretación, se basa también en conceptos expresados por los docentes, sobre estudiantes que son reconocidos como muy capaces intelectualmente, pero cuyas conductas de irresponsabilidad (incumplimiento en la entrega de trabajos, inasistencia, retrasos, etc.) no permiten calificarlos como buenos. El caso es similar para aquellos estudiantes del nivel medio o regular, que teniendo dificultades de tipo cognitivo, ya que los docentes deben a menudo hacerles tutorías individuales a través del $\mathrm{PAOE}^{7}$, logran buenas notas gracias a su dedicación, motivación y sentido de responsabilidad.

Similar situación se presenta si se comparan los clasificados en los niveles regular y bajo. Si bien en el primero se encuentran generalmente estudiantes con promedios de 4.0 a 4.4, los hay también de 3.3 a 3.9. En el nivel bajo, los hay con promedios de 3.9 e incluso 4.1. Este resultado puede haber sido provocado por lo que los docentes denominaron, entre las cualidades personales, como el ser dependientes, ya que algunos estudiantes buscan realizar los trabajos en grupo, con compañeros de mayor nivel intelectual, con el fin de obtener una mejor nota.

7 Programa de atención y orientación al estudiante 
A nivel general, también es significativo el número de estudiantes de cada categoría de evaluación cualitativa. Un 43.7 \% fue calificado como bueno, un $35.4 \%$ como regular o de nivel medio y un 20.8 \% como bajo. Lo llamativo de este resultado es que normalmente se espera que la mayoría se ubique en el nivel medio y las minorías en los dos extremos. Valdría entonces la pena contrastar estos resultados con otras variables que podrían estar afectando estas calificaciones, tanto positiva como negativamente, tales como, el enfoque y estrategias de enseñanza y las formas de evaluación, lo que podría ser objeto de futuras investigaciones. Por el momento, en lo que a este informe se refiere, los resultados relativos al rendimiento académico, fueron contrastados con los estilos de aprendizaje de los estudiantes, lo cual es objeto del análisis que a continuación se presenta, con base en la

Tabla 2:

Estilos de aprendizaje

\begin{tabular}{ccccccccc}
\hline \hline Nivel & \multicolumn{7}{c}{ Estilos de aprendizaje } \\
\cline { 2 - 9 } & $\mathbf{A}$ & $\mathbf{R}$ & $\mathbf{S n}$ & $\mathbf{I}$ & $\mathbf{V i}$ & $\mathbf{V r}$ & $\mathbf{S c}$ & $\mathbf{G}$ \\
\hline \multirow{2}{*}{ Bueno } & 15 & 6 & 12 & 9 & 11 & 10 & 12 & 9 \\
\hline \hline \multirow{2}{*}{ Medio } & $71.4 \%$ & $28.6 \%$ & $57 \%$ & $43 \%$ & $52.4 \%$ & $47.6 \%$ & $57 \%$ & $43 \%$ \\
\cline { 2 - 9 } & 76 & 4 & 9 & 8 & 13 & 4 & 9 & 8 \\
\cline { 2 - 9 } Bajo & 8 & $23.5 \%$ & $53 \%$ & $47 \%$ & $76.5 \%$ & $23.5 \%$ & $53 \%$ & $47 \%$ \\
\cline { 2 - 9 } & $80 \%$ & $20 \%$ & $60 \%$ & $40 \%$ & $80 \%$ & $20 \%$ & $50 \%$ & $50 \%$ \\
\hline \hline
\end{tabular}

Siguiendo el modelo de Felder y Silverman, la oposición en categorías bipolares, sugiere una interpretación de la Tabla 2 en ese mismo sentido. Se observa entonces que en la primera pareja (activo/reflexivo), los mayores porcentajes corresponden al estilo activo (bueno $71.4 \%$, regular $76.5 \%$ y bajo $80 \%$ y los menores al reflexivo (bueno $28.6 \%$, regular $23.5 \%$ y bajo $20 \%)$. Por otra parte, en la categoría activo, tales porcentajes aumentan a medida que se baja de nivel (los de nivel bueno son menos activos que los regulares y éstos lo son menos que los del nivel bajo). En la categoría reflexivo, por el contrario, éstos disminuyen (los de nivel bueno son más reflexivos que los del regular y éstos lo son más que los de nivel bajo). 
Estos resultados sugieren que falta un mayor equilibrio entre estos dos estilos de aprender; el predominio del estilo activo hace que los estudiantes no sean dados a pensar y a organizar su mente para retener más la información conceptual por haber reflexionado sobre lo que ésta propone. Esta apreciación coincide con el hecho de que los enfoques actuales de enseñanza del inglés proponen un mayor número de componentes que exigen la participación dinámica de los estudiantes, si se compara con las actividades lectura y escritura reflexivas, críticas o argumentativas. Además, la mayoría de los docentes de inglés optan por ofrecer previamente las explicaciones (gramática, sintaxis, etc.), en lugar de dejar que los estudiantes infieran las reglas con base en reflexiones.

En el caso de la segunda pareja bipolar (sensitivo/intuitivo), los resultados son similares a la pareja anterior: los estudiantes son más sensitivos que intuitivos. Es decir, prefieren guiarse por procedimientos preestablecidos y se les facilita aprender si llevan todo a la práctica, características que se anteponen al tratamiento de las abstracciones, la creatividad y la innovación. Es de anotar, sin embargo, que en el sentido vertical de cada categoría de estilo de aprendizaje, no se presenta tan estrictamente el fenómeno de aumento o disminución de los porcentajes según las categorías cualitativas, como en el caso del binomio activo/ reflexivo. Como en las categorías precedentes, los manuales utilizados para las clases de inglés, son generadores de esta situación, ya que aportan cuadros, reglas, ejercicios, etc. que encierran al estudiante en procedimientos predeterminados y reglas preestablecidas que el estudiante debe aprender y seguir. Las evaluaciones propuestas por los docentes también giran alrededor de tales procedimientos.

El comportamiento es también el mismo para la tercera categoría bipolar (visual/verbal), ya que la tendencia es la de ser más visuales que verbales. Esto se acentúa a medida que baja el nivel (bueno $52 \%$, regular $76 \%$, bajo $80 \%$ ), para el caso del estilo visual, y disminuye para el estilo verbal (bueno $47.6 \%$, regular $23.5 \%$, bajo $20 \%$ ). Lo anterior implica que a los estudiantes se les facilita aprender cuando la información se presenta en forma de gráficas, imágenes, dibujos, entre otras ilustraciones. Por el contrario, tienen dificultades para el tratamiento de la información de tipo verbal, lo que se refleja en las dificultades que generalmente se tienen en Colombia para los procesos de lectura y escritura en el nivel universitario. En el caso concreto del inglés, nuevamente acudimos a los factores del texto guía y el docente. El primero contiene cantidades 
significativas de imágenes y cuadros; el segundo debe añadir a esas ilustraciones, otras como videos, láminas, afiches, etc. so pena de ver disminuido el interés de sus alumnos en sus clases.

La cuarta pareja bipolar presentó también la misma tendencia: el estilo secuencial predomina sobre el global. Esto implica que los estudiantes aprenden más fácilmente cuando la información se les presente paso a paso y pueden ir relacionado cada nueva etapa con la anterior, pero tienen dificultades para captar la complejidad o múltiples dimensiones de un problema, como un todo global. Paradójicamente, en el sentido vertical de la columna, la situación fue totalmente opuesta: para el estilo secuencial los porcentajes bajan a medida que disminuye el nivel (bueno $57 \%$ ) regular $53 \%$ y bajo $50 \%$ ); mientras que en la categoría de estilo global ocurre lo opuesto ya que los porcentajes aumentan a medida que baja el nivel (bueno $43 \%$, regular $47 \%$, bajo $50 \%$ ). A pesar de que los nuevos enfoques preconizan el trabajo por proyectos globales y complejos, docentes y los conceptores de textos de inglés parecen estar muy apegados al concepto estructuralista de la lengua que sugiere su estudio secuencial y ordenado en categorías que van de lo fácil a lo difícil, de lo simple a lo complejo.

\section{Conclusiones}

Determinar el estilo de aprendizaje de una persona es sin duda una tarea de una dimensión psicológica muy compleja, que requiere de muchas y diversas pruebas, además de la observación y análisis de un proceso que por sus características es predominantemente interno al ser. Sin embargo, estudios parciales como el que aquí se presenta, constituyen un acercamiento válido a este complejo fenómeno psicológico y se convierten en una herramienta que puede ser utilizada para el mejoramiento, tanto de los procesos de aprendizaje, como de los de enseñanza.

Tener la información, como es el caso de los resultados antes analizados, de que los estudiantes tienen estilos de aprendizaje predominantemente activos, sensitivos, visuales y secuenciales, es una señal que puede ser explotada en dos sentidos. En primera instancia, los docentes pueden darse a la tarea de facilitar el aprendizaje, a través del uso de estrategias pedagógicas basadas en información que sea presentada acorde con esos estilos; en segundo lugar, tales resultados pueden encender la voz de alarma acerca de la necesidad de incentivar procesos de aprendizaje que 
promuevan procesos mentales que favorezcan el aprendizaje de carácter reflexivo, intuitivo, verbal y global. Estas acciones estarían orientadas a la búsqueda de un equilibrio en el estilo de aprendizaje de los estudiantes, dado que lo ideal es que exista un balance entre cada categoría bipolar y no que una predomine sobre la otra.

En relación con el rendimiento académico, el análisis de los resultados dio a conocer que las diferencias o distancias, en términos cuantitativos, no son tan grandes entre un nivel y el siguiente. Esto indica que la medición del aprendizaje a través de un indicador numérico, no está reflejando fielmente la calidad de los resultados logrados por los estudiantes, o que éstos no son evaluados de manera que se pueda apreciar claramente su verdadero nivel. También podría inferirse que este tipo de evaluación, no está teniendo en cuenta algunos de los componentes cualitativos del proceso, como son la actitud de los estudiantes frente a las actividades de clase y a los trabajos asignados, o sus características personales reflejadas en su sentido de responsabilidad, motivación e interés. Esta consideración se refleja en el hecho de que los docentes, en la sesión de grupo focal, fueron mucho más exigentes al calificar a sus estudiantes cualitativamente, de lo que se refleja en los promedios de notas obtenidos por éstos.

La anterior consideración, conduce a plantear la necesidad de implementar cambios en las estrategias de enseñanza del inglés, así como un ajuste en los criterios y formas de evaluación. Si a esto se sumara, la propuesta antes planteada, en el sentido en que es también necesario tratar de que haya un mayor equilibrio entre las categorías bipolares de estilos de aprendizaje, seguramente la situación cambiaría de manera positiva y favorable a un mejor logro de los objetivos de aprendizaje planteados para la formación de los futuros docentes, egresados del programa de Licenciatura en Lenguas Modernas. El desarrollo de estrategias didácticas orientadas hacia los diversos estilos de aprendizaje, sería el medio más adecuado para lograr el equilibrio antes señalado, lo que repercutiría en un mejor rendimiento académico.

\section{Referencias}

Alcaide, Margarita (2009) Influencia del rendimiento académico y auto concepto en hombres y mujeres. Revista Reid 2. pp 27-44. Disponible en http://www.ujaen.es/revista/reid/revista/n2/REID2art2.pdf 
Arvizu, B. (2010). Estilos de aprendizaje. Ensenada (Baja California): U. Estatal de estudios pedagógicos pp. 6-7. Disponible en: http:// es.scribd.com/doc/43208451/Estilos-de-Aprendizaje-Doc-Final.

Cisneros, A. (2004) Manual de estilos de aprendizaje: material autoinstruccional para docentes y orientadores educativos. México: SEP (Secretaría de Educación Pública). Disponible en http://www. slideshare.net/peques1718c/manual-de-estilos-de-aprendizaje.

Cloninger, S. (2003). Teorías de la Personalidad. México: Pearson Education.

García Cué, J. L. (2006). Aprendizaje. Disponible en http://www.jlgcue. es/aprendizaje.htm

Gottfredson, L. (2004). "Intelligence: Is it the epidemiologists' elusive "fundamental cause" of social class inequalities in health?" Journal of Personality and Social Psychology. Vol. 86 (1), pp. 174 - 199.

Guanipa, M. y Mogollón, E. (2006): “Estilos de aprendizaje y estrategias cognitivas en estudiantes de ingeniería". Revista Ciencias de la Educación 1 (27), pp. 11- 27. Disponible en http://servicio.bc.uc. edu.ve/educacion/revista/volIn27/27-1.pdf

Hernández, L. (2004). "Estilos de aprendizaje predominantes en una población representativa de estudiantes de inglés del CELE de la UNAM". Espéculo: Revista de estudios literarios. No. 27 Madrid: Universidad Complutense de Madrid. Disponible en http://www. ucm.es/info/especulo/numero27/estilosa.html

Hernández, A. y Cardona, A. (2008). Estilos y Estrategias de Aprendizaje en el rendimiento académico de los alumnos del área de Inglés de la Licenciatura en Lenguas Modernas de la Universidad de La Salle. Repositorio Institucional Unisalle. Disponible en

http://repository.lasalle.edu.co/bitstream/10185/1669/1/T85.08\%20H431e. pdf

Loaiza, N. y Guevara, A. (2012). Los estilos de aprendizaje: una propuesta pedagógica para optimizar la enseñanza de las lenguas extranjeras en La licenciatura en lenguas modernas de la Universidad del Quindío. Revista de Investigaciones. Disponible en http://www. uniquindio.edu.co/uniquindio/revistainvestigaciones/adjuntos/ pdf/13cc_Art\%203\%20[OK].pdf 
Manzano, M. e Hidalgo, E. (2009). “Estilos de aprendizaje, estrategias de lectura y su relación con el rendimiento académico de la lengua extranjera". En Redalyc No. 12 pp. 123-150 Universidad Nacional de Educación a Distancia, España. Disponible en http://redalyc. uaemex.mx/pdf/706/70611919007.pdf

Ovejero, A. (2007). Las Relaciones Humanas. Psicología Social Teórica y Aplicada. Madrid: Biblioteca Nueva - Psicología Universidad de Madrid.

Pérez, A. y Gimeno, J. (1992). Comprender y transformar la enseñanza. Madrid: Morata pp. 34-57. Disponible en http://books.google.com. $\mathrm{co} /$ books? id =J6OTlHYpkm0C\&printsec $=$ frontcover $\& \mathrm{hl}=$ es \&source $=g b s \_g e \_s u m m a r y \_r \& c a d=0 \# \mathrm{v}=$ onepage $\& \mathrm{q} \& \mathrm{f}=$ false

Salas, R. (2008). Estilos de aprendizaje a la luz de la Neurociencia. Bogotá: Magisterio.

Terradez, M. (2007). Los estilos de aprendizaje aplicados a la enseñanza del español como lengua extranjera. Revista Foro de Profesores de ELE vol. 3 pp. 227-230. Disponible en http://www.uv.es/foroele/ foro3/Terradez.pdf 


\section{Descripción de categorías por niveles de rendimiento académico. Grupo focal docentes}

\begin{tabular}{|c|c|c|c|}
\hline \multirow[t]{2}{*}{ Categorías } & \multicolumn{3}{|c|}{ Niveles } \\
\hline & Bueno & Medio & Bajo \\
\hline Personalidad & $\begin{array}{l}\text { Personas } \\
\text { responsables, } \\
\text { comprometidas } \\
\text { con sus estudios, } \\
\text { auto-motivadas, } \\
\text { disciplinadas, } \\
\text { colaboradoras. } \\
\text { Generalmente se } \\
\text { visionan como } \\
\text { docentes, tienen } \\
\text { claridad sobre } \\
\text { su vocación. }\end{array}$ & $\begin{array}{l}\text { Son estudiantes } \\
\text { dependientes } \\
\text { del docente } \\
\text { Se limitan } \\
\text { solamente a lo } \\
\text { que se les pide, no } \\
\text { se preocupan por } \\
\text { saber más; no se } \\
\text { esmeran por buscar } \\
\text { una solución } \\
\text { No demuestran } \\
\text { tanto interés, son } \\
\text { fluctuantes por falta } \\
\text { de disciplina. Esta } \\
\text { sólo responde a } \\
\text { razones académicas } \\
\text { o familiares. No son } \\
\text { tan extrovertidos, } \\
\text { son pasivos y } \\
\text { conformistas }\end{array}$ & $\begin{array}{l}\text { Son incumplidos } \\
\text { (inasistencia, } \\
\text { retrasos); no les } \\
\text { interesa dar excusas } \\
\text { por su inasistencia. } \\
\text { Son irresponsables } \\
\text { y desmotivados } \\
\text { No tienen ninguna } \\
\text { visión frente a lo } \\
\text { que quieren. } \\
\text { Son personas que } \\
\text { siempre están solas } \\
\text { No les gusta } \\
\text { el estudio, ni } \\
\text { la lectura }\end{array}$ \\
\hline Actitud & $\begin{array}{l}\text { Son estudiantes } \\
\text { atentos, activos, } \\
\text { colaboradores, } \\
\text { ayudan a sus } \\
\text { compañeros, les } \\
\text { gusta el trabajo } \\
\text { en equipo, } \\
\text { participan } \\
\text { en clase. }\end{array}$ & $\begin{array}{l}\text { Son personas } \\
\text { tímidas y se cohíben } \\
\text { fácilmente. Tienen } \\
\text { autoestima y perfil } \\
\text { de bajo nivel. No } \\
\text { toman riesgos, no se } \\
\text { atreven a intervenir, } \\
\text { tratan de pasar } \\
\text { desapercibidos. } \\
\text { Son dependientes, } \\
\text { por conveniencia. } \\
\text { Su nivel de } \\
\text { compromiso es bajo, } \\
\text { sólo les interesa la } \\
\text { nota. Algunos hacen } \\
\text { mucho esfuerzo } \\
\text { pero no producen, } \\
\text { porque no tienen la } \\
\text { actitud adecuada }\end{array}$ & $\begin{array}{l}\text { Demuestran } \\
\text { desmotivación ante } \\
\text { cualquier actividad. } \\
\text { Rechazan el trabajo, } \\
\text { no hacen tareas, } \\
\text { suelen copiarlas } \\
\text { de sus compañeros } \\
\text { o de internet. } \\
\text { No se interesan } \\
\text { en entender } \\
\text { Son mediocres, } \\
\text { perezosos, amantes } \\
\text { de la vida fácil. Son } \\
\text { como parásitos pues } \\
\text { siempre buscan } \\
\text { trabajar con alguien } \\
\text { para no hacer } \\
\text { nada. No poseen } \\
\text { talento para lo que } \\
\text { están estudiando }\end{array}$ \\
\hline
\end{tabular}




\begin{tabular}{|c|c|c|c|}
\hline \multirow[t]{2}{*}{ Categorías } & \multicolumn{3}{|c|}{ Niveles } \\
\hline & Bueno & Medio & Bajo \\
\hline $\begin{array}{c}\text { Capacidades } \\
\text { mentales }\end{array}$ & $\begin{array}{l}\text { Son inteligentes, } \\
\text { tienen buena } \\
\text { memoria y } \\
\text { capacidades de } \\
\text { comprensión } \\
\text { y habilidades } \\
\text { previas en } \\
\text { lacto escritura. } \\
\text { Son críticos, es } \\
\text { decir exponen } \\
\text { sus puntos de } \\
\text { vista sobre las } \\
\text { situaciones y } \\
\text { temas de estudio. }\end{array}$ & $\begin{array}{l}\text { No tienen disciplina } \\
\text { en el estudio. } \\
\text { Son lentos en } \\
\text { el aprendizaje, } \\
\text { necesitan refuerzos } \\
\text { y trabajo adicional. } \\
\text { No profundizan, } \\
\text { no hacen análisis. } \\
\text { Sus puntos de vista } \\
\text { no son claros. }\end{array}$ & $\begin{array}{l}\text { Son perezosos } \\
\text { y lentos en el } \\
\text { aprendizaje } \\
\text { hacer resúmenes; } \\
\text { Se les dificulta } \\
\text { hacer resúmenes, } \\
\text { dar su punto de } \\
\text { vista frente a } \\
\text { algún tema. } \\
\text { No saben defender } \\
\text { sus ideas } \\
\text { No proponen nada } \\
\text { nuevo o diferente } \\
\text { para la clase. } \\
\text { Lo que se les da, } \\
\text { para ellos es más } \\
\text { que suficiente }\end{array}$ \\
\hline
\end{tabular}

